MKPH-T-93-04, IU/NTC 92-20

March 24, 1993

\title{
Nuclear Shadowing in a Parton Recombination Model
}

\author{
S. KUMANO * \\ Institut für Kernphysik, Universität Mainz \\ 6500 Mainz, Germany \\ and \\ Nuclear Theory Center, Indiana University \\ Bloomington, Indiana 47408, U.S.A.
}

\begin{abstract}
Deep inelastic structure functions $F_{2}^{A}(x)$ are investigated in a $Q^{2}$ rescaling model with parton recombination effects. We find that the model can explain experimentally measured $F_{2}^{A}(x)$ structure functions reasonably well in the wide Bjorken $-x$ range $(0.005<x<0.8)$. In the very small $x$ region $(x<0.02)$, recombination results are very sensitive to input sea-quark and gluon distributions.
\end{abstract}

* address after April 1, 1993: Department of Physics, Saga University, Saga 840, Japan.

submitted to Phys. Rev. C

PACS numbers: $13.60 . \mathrm{Hb}, 25.30 .-\mathrm{c}$ 


\section{Introduction}

Several years have passed since the discovery of nuclear modifications in structure functions $F_{2}(x)$ by the European Muon Collaboration ("old" EMC effect) [1]. In spite of initial expectation of an explicit quark signature in nuclear physics, it is still not clear whether the effect originates in nucleon substructures or just in nuclear physics. There are several proposed models for explaining the EMC effect. Some models tried to interpret it by explicit quark effects, e.g. $Q^{2}$ rescaling models [2] and six-quark bag models. Others investigated models based on conventional nuclear physics, such as nuclear binding and pion excess. For details of these models, we suggest that interested readers look at summary papers in Ref. 3. Although there are still experimental activities [4] for investigating these different models, it is rather difficult to discriminate among these models.

Most of these investigations discuss a "global" EMC effect in the sense that the effect is averaged over all constituents in a nucleus. However, it is interesting to investigate possible semi-inclusive or semi-exclusive processes for finding a "local" EMC effect [5] and possible relations between a local gluonic EMC effect and the $J / \psi$ suppression [6].

Considering the fact that the average nucleon separation in nuclei is $2.2 \mathrm{fm}$ and the nucleon diameter is $1.8 \mathrm{fm}$, we expect that nucleons in nuclei could overlap strongly. If a multiquark cluster is formed in a nucleus due to this overlap, the confinement radius for such a quark should be different from the one in a free nucleon. Using this kind of simple picture in a small $Q^{2}$ region and the $Q^{2}$-evolution equation to compare with experimental data at large $Q^{2}$, we obtain a simple prescription for the nuclear structure function $F_{2}^{A}\left(x, Q^{2}\right)$ [2]. It is related to the nucleon structure function by a simple $Q^{2}$ rescaling, $F_{2}^{A}\left(x, Q^{2}\right)=F_{2}^{N}\left(x, \xi_{A} Q^{2}\right)$, where $\xi_{A}$ is called as rescaling parameter. Although the simple size change could be too simple to explain many details of nuclear physics [7], it is a useful effective model in explaining deep inelastic data in the medium $x$ region.

The New Muon Collaboration (NMC) [8] and the Fermilab E665 collaboration [9] recently measured accurately the structure functions $F_{2}(x)$ at very small $x$. These data as well as EMC data [10] provide an opportunity for investigating physics details in the shadowing region $(x<0.1)$. The shadowing means that central constituents are shielded due to surface constituents, hence the cross section behaves like $A^{\alpha}(\alpha<1)$. There are 
different ideas for explaining the shadowing phenomena: vector meson dominance [11], parton recombination [12-17], $q \bar{q}$ fluctuations of the virtual $\gamma[18]$, pomeron dominance [19] (with Pauli blocking [20]), and hybrid models [21].

At first, there are models based on the traditional idea, the vector meson dominance model [11]. A virtual photon transforms into vector meson states $(\rho, \omega, \phi)$, which then interacts with a target nucleus. The propagation length of the hadronic $(\mathrm{H})$ fluctuations is given by $\lambda \approx 1 /\left|E_{H}-E_{\gamma}\right|=2 \nu /\left(M_{H}^{2}+Q^{2}\right) \approx 0.2 / x$ fm. For $x<0.1$, the propagation length $(>2 \mathrm{fm})$ exceeds the average nucleon separation $(2.2 \mathrm{fm})$ in nuclei and the shadowing takes place due to multiple scatterings. For example, the vector meson interacts elastically with a surface nucleon and then interacts inelastically with a central nucleon. Because this amplitude is opposite in phase to a one-step amplitude for an inelastic interaction with the central nucleon, the nucleon sees a reduced hadronic flux (namely the shadowing).

The parton recombination model [12-17] has been investigated as a mechanism for explaining the shadowing within the framework of a quark-parton model. In an infinite momentum frame, the average longitudinal nucleon separation in a Lorentz contracted nucleus is $L=(2.2 \mathrm{fm}) M_{A} / P_{A}=(2.2 \mathrm{fm}) m_{N} / p_{N}$, and the longitudinal localization size of a parton with momentum $x p_{N}$ is $\Delta L=1 /\left(x p_{N}\right)$. If the parton dimension $(\Delta L)$ exceeds the average nucleon separation $(L)$, partons from different nucleons could interact (we call this parton recombination or parton fusion) significantly and the shadowing could occur due to processes in Figs. 1b, 1c, 1e, and 1f. Partons with momentum fraction $x$ are lost in these processes, so that their contributions are negative $\Delta F_{2}(x)<0$ (shadowing). However, $\Delta F_{2}(x)$ depends much on input sea-quark and gluon distributions as we find in sections 3 and 4. A relevant $x$ region for the shadowing is obtained by using $L<\Delta L$ and we find $x<0.1$. Even though the recombinations could produce shadowing type effects in the small $x$ region, they are very small compared with experimental shadowings if they are calculated at $Q^{2}=5 \mathrm{GeV}^{2}$. On the other hand, modifications of $F_{2}(x)$ due to recombination effects on gluon distributions are large [17]. Details of numerical results are discussed in section 3 .

Because the recombination results by Close, Qiu, and Roberts [17] are not compared in detail with the recent experimental data, we investigate whether the model can explain 
the EMC, NMC, and E665 shadowing data in this investigation. Furthermore, we study whether the $Q^{2}$ rescaling model combined with parton recombination effects [14,15] can explain the $F_{2}(x)$ structure functions in the whole Bjorken- $x$ range [16].

Earlier comparisons with experimental data have been made in similar parton pictures [15]. Let us clarify differences from and advantages over the previous investigations. (1) Covolan, Predazzi, and others [15] used the Qiu's parametrization [13] for the shadowing and combined its results with the rescaling. We note that the $Q^{2}$ evolution is well investigated in Ref. 13; however, the $x$ dependence is rather assumed. Therefore, we cannot have a dynamically consistent picture by using the shadowing-parametrization with the rescaling. The $x$ dependence of the shadowing was later investigated in Ref. 17. Using this shadowing picture combined with the rescaling, we could possibly obtain a dynamicallyconsistent parton model in the wide $x$-range. This is the purpose of our investigation. (2) We study $Q^{2}$ evolution effects in the combined model. (3) We investigate effects of input sea-quark and gluon distributions on the shadowing. (4) Improved experimental data became available by the NMC and E665.

\section{Parton Recombinations}

If a nucleon is in a nucleus, parton distributions are modified because of the existence of neighboring nucleons. Partons from different nucleons could interact with each other, and the interactions become important, especially in the shadowing region. Processes contribute to modifications in a quark distribution $q(x)$ are shown in Fig. 1. For example, Fig. 1a indicates that a quark from the nucleon 1 fuses with a gluon from the nucleon 2 and produces a quark with momentum $x$. Because the process creates a quark with the momentum $x$, this is a positive contribution to the quark distribution at $x$. There are five other contributions as shown in Fig. 1. In general, a modification of a parton distribution $p_{3}\left(x_{3}\right)$, due to the process of producing the parton $p_{3}$ with the momentum $x_{3}$ by a fusion of partons $p_{1}$ and $p_{2}$, is given by [17]

$$
\Delta p_{3}\left(x_{3}\right)=K \int d x_{1} d x_{2} p_{1}\left(x_{1}\right) p_{2}\left(x_{2}\right) \Gamma_{p_{1} p_{2} \rightarrow p_{3}}\left(x_{1}, x_{2}, x_{3}=x_{1}+x_{2}\right) \delta\left(x, x_{1}, x_{2}\right)
$$

where $K$ is given by $K=9 A^{1 / 3} \alpha_{s} /\left(2 R_{0}^{2} Q^{2}\right)$ [22]. Nuclear radius $R$ is $R=R_{0} A^{1 / 3}$ with 
$R_{0}=1.1 \mathrm{fm}[23]$ and the strong-interaction coupling constant is $\alpha_{s}\left(Q^{2}\right)=4 \pi /\left[\beta_{0} \ln \left(Q^{2} / \Lambda^{2}\right)\right]$ with $\beta_{0}=11-2 N_{f} / 3, N_{f}=3$. The momentum conserving $\delta$ function is given by $\delta\left(x-x_{1}-x_{2}\right)$ for Figs. 1a and 1d and $\delta\left(x-x_{1}\right)$ for Figs. 1b, 1c, 1e, and 1f. The parton fusion function $\Gamma\left(x_{1}, x_{2}, x_{3}\right)$ is a probability for producing a parton $p_{3}$ with momentum $x_{3}$ by a fusion of partons $p_{1}$ and $p_{2}$ with momenta $x_{1}$ and $x_{2}$ respectively. Four possible parton fusion processes are shown in Fig. 2. It is related to a splitting function $P_{p_{1} p_{3}}(z)$ in the Altarelli-Parisi equation [24] by

$$
\Gamma_{p_{1} p_{2} \rightarrow p_{3}}\left(x_{1}, x_{2}, x_{3}\right)=\frac{x_{1} x_{2}}{x_{3}^{2}} P_{p_{1} \leftarrow p_{3}}\left(\frac{x_{1}}{x_{3}}\right) C_{p_{1} p_{2} \rightarrow p_{3}} .
$$

The splitting function $P_{p_{1} p_{3}}(z)$ is the probability of finding the parton $p_{1}$ with fraction $z$ of the parent momentum in the parton $p_{3} . C_{p_{1} p_{2} \rightarrow p_{3}}$ is the ratio of color factors in processes $p_{1} p_{2} \rightarrow p_{3}$ and $p_{3} \rightarrow p_{1} p_{2}$. For example, $C_{q G \rightarrow q}=\sum_{(l, a), k}^{-}\left(t_{k l}^{a}\right)^{*} t_{k l}^{a} / \sum_{(k), l, a}^{-}\left(t_{l k}^{a}\right)^{*} t_{l k}^{a}=$ $\left[C_{F} /\left(N_{C}^{2}-1\right)\right] / C_{F}$, where averages are taken over initial color indices ( $k$ in the denominator, $l$ and $a$ in the numerator). $t_{l k}^{a}$ is given by the $S U(3)_{c}$ Gell-Mann matrix by $t_{l k}^{a}=\lambda_{l k}^{a} / 2$, and $C_{F}$ is $C_{F}=\left(N_{C}^{2}-1\right) /\left(2 N_{C}\right)[25]$. In this way, the color factors are calculated as:

$$
\begin{array}{ll}
C_{q G \rightarrow q}=\frac{1}{N_{C}^{2}-1}=\frac{1}{8} \quad, & C_{q \bar{q} \rightarrow G}=\frac{C_{F}}{N_{C} T_{F}}=\frac{8}{9}, \\
C_{G q \rightarrow q}=\frac{T_{F}}{N_{C} C_{F}}=\frac{1}{8} & ,
\end{array}
$$

where $T_{F}=1 / 2$ and $N_{C}=3$. Using the above results and splitting functions in Ref. 24, we obtain the parton fusion functions:

$$
\begin{aligned}
& \Gamma_{q G \rightarrow q^{\prime}}\left(x_{1}, x_{2}, x_{3}\right)=\frac{1}{6} z\left(1+z^{2}\right), \\
& \Gamma_{q \bar{q} \rightarrow G}\left(x_{1}, x_{2}, x_{3}\right)=\frac{4}{9} z(1-z)\left[z^{2}+(1-z)^{2}\right], \\
& \Gamma_{G q \rightarrow q^{\prime}}\left(x_{1}, x_{2}, x_{3}\right)=\frac{1}{6}(1-z)\left[1+(1-z)^{2}\right], \\
& \Gamma_{G_{1} G_{2} \rightarrow G_{3}}\left(x_{1}, x_{2}, x_{3}\right)=\frac{3}{4} z(1-z)\left[\frac{z}{1-z}+\frac{1-z}{z}+z(1-z)\right],
\end{aligned}
$$

where $x_{3}=x_{1}+x_{2}$ and $z \equiv x_{1} / x_{3}$. 
Using these fusion functions, we calculate modifications of quark distributions in nuclei for processes shown in Fig. 1. Explicit expressions for the modifications $\Delta q_{i}(x)$ are shown in Appendix (Eq. (A1)). From Eq. (A1) and a similar expression for $\Delta \bar{q}_{i}(x)$, we obtain recombination contributions to the structure function $F_{2}(x)$ in Eqs. (A2.1)-(A2.4). Direct recombination effects on the EMC effect are given by the ratio

$$
R_{A}(\text { recombination })=1+\frac{\Delta F_{2}^{A}\left(x, Q^{2}\right)}{F_{2}^{D}\left(x, Q^{2}\right)},
$$

where $\Delta F_{2}^{A}\left(x, Q^{2}\right)$ is calculated by using Eqs. (A2.1) - (A2.4) with input parton distributions at $Q^{2}$. The most important physics for the shadowing in the recombination model is the effect due to modifications in gluon distributions. Recombination effects on the gluon distribution are given in Eqs. (A3.1)-(A3.4). Calculating these equations, we find that the recombination mechanism produces large shadowing effects on the gluon distribution $[17]$.

\section{Gluon-shadowing effects on $\mathbf{F}_{2}^{\mathrm{A}}(\mathbf{x})$}

\subsection{An approximate way to take into account the gluon shadowing}

Gluons and quarks are coupled to each other and their distributions are related by the Altarelli-Parisi equation. Because of the large modifications in $G(x<0.1)$ due to the recombinations, we expect that sea-quark distributions are also affected by the gluon modifications. In the very small $x$ region, the Altarelli-Parisi equation is dominated by gluon dynamics, and it can be solved analytically. As a result, there is a relationship between a sea quark and gluon distributions [26]: $x q_{i}^{\text {sea }}(x)=-\frac{x}{12} \frac{\partial}{\partial x}[x G(x)]$, where $i=\mathrm{u}$, $\mathrm{d}$, or s. Using this relation, we obtain a contribution to $F_{2}(x)$ from the modification in the gluon distribution:

$$
\delta F_{2}\left(x, Q^{2}\right)=-\theta\left(x_{0}-x\right) \frac{4}{3} \frac{x}{12} \frac{\partial}{\partial x}\left[x \Delta G\left(x, Q^{2}\right)\right]
$$

where $x \Delta G\left(x, Q^{2}\right)$ is given in Eqs. (A3.1)-(A3.4), and a step function $\theta\left(x_{0}-x\right)$ is introduced because the relation is valid only at very small $x$. It is defined by $\theta\left(x_{0}-x\right)=1$ (for $x<x_{0}$ ) or 0 (for $x>x_{0}$ ). We should note that this is a crude estimate of the gluonic 
contribution. To be precise, the recombination calculations are done at small $Q^{2}$, then the results are evolved to the $Q^{2}$ region where experiments were done, by using a nuclearmodified Altarelli-Parisi equation [13]. This approach is discussed in section 4. Combining all the contributions, we obtain nuclear structure functions $F_{2}^{A}(x)$ calculated in our parton model (the $Q^{2}$ rescaling model with parton recombination effects):

$$
\frac{F_{2}^{A}\left(x, Q^{2}\right)}{F_{2}^{D}\left(x, Q^{2}\right)}=\frac{\tilde{F}_{2}^{A}\left(x, Q^{2}\right)+\Delta F_{2}^{A}\left(x, Q^{2}\right)+\delta F_{2}^{A}\left(x, Q^{2}\right)}{F_{2}^{D}\left(x, Q^{2}\right)}
$$

where $\tilde{F}_{2}^{A}$ is given by $\tilde{F}_{2}^{A}\left(x, Q^{2}\right)=F_{2}^{N}\left(x, \xi_{A} Q^{2}\right)$ in the rescaling model.

\subsection{Results by using the analytical solution at small $x$}

We calculate nuclear structure functions and investigate whether our theoretical results are compatible with experimental data [27] by a SLAC group [28], EMC [10,29], NMC [8], and E665 [9]. It is interesting to investigate not only the shadowing region at small $x$ but also the large $x$ region, which is traditionally called "nucleon-Fermi-motion" part.

In evaluating equations in Appendix, we first assume that a leak-out parton is a sea quark or a gluon and that the momentum cutoff function [17,30] for this parton is taken as $w(x)=\exp \left(-m_{N}^{2} z_{0}^{2} x^{2} / 2\right)$, namely $q^{*}(x)=w(x) q_{\text {sea }}(x), \bar{q}^{*}(x)=w(x) \bar{q}(x)$, and $G^{*}(x)=w(x) G(x)$. Input parton distributions are given by a recent parametrization by Martin, Roberts, and Stirling (MRS) [31] or by Kwiecinski, Martin, Roberts, and Stirling (KMRS) [32]. $Q^{2}=5$ (or 4) $\mathrm{GeV}^{2}$ is used in the input parton distributions and for calculating $K$ in Eq. (1). The cutoff function $w(x)$ supposedly takes into account effects of bound partons and it is shown as the function of $x$ in Fig. 3. As discussed in the introduction, $x=0.1$ corresponds to the length scale $2 \mathrm{fm}$, the average nucleon separation in nuclei. Hence, we expect that only partons $p^{*}$ with $x_{\sim}^{<} 0.1$ could participate in the recombinations. We then find in Fig. 3 that an appropriate choice is $z_{0}=2-3 \mathrm{fm}$. We show theoretical results with $z_{0}=2 \mathrm{fm}$ in this section; some $z_{0}$ dependencies are discussed in section 4.3. $x_{0}$ in Eq. (6) is taken as $x_{0}=0.1$. We do not show the ratio in Eq. (7) in the $x$ range $0.1<x<0.2$ because physics is not well described by the crude estimate in Eq. (6) for taking into account the gluonic modifications. This $x$ region is investigated by 
a more-complete $Q^{2}$-evolution picture in section 4. The QCD scale parameter $\Lambda$ in $\alpha_{s}\left(Q^{2}\right)$ is taken as $\Lambda=0.2 \mathrm{GeV}$.

In our theoretical analysis, $\mathrm{Ag}$, $\mathrm{Sn}$, and Xe targets are assumed as ${ }^{107} \mathrm{Ag},{ }^{118} \mathrm{Sn}$, and ${ }^{131}$ Xe nuclei. We take the rescaling parameters in Table II of the Close, Jaffe, Roberts, and Ross paper [2]. They are $\xi_{A}=1.43\left({ }^{4} \mathrm{He}\right), 1.60\left({ }^{12} \mathrm{C}\right), 1.86\left({ }^{40} \mathrm{Ca}\right), 2.17\left({ }^{107} \mathrm{Ag}\right)$, and 2.24 $\left({ }^{118} \mathrm{Sn}\right) . \xi_{A}=2.24$ is assumed for the ${ }^{131} \mathrm{Xe}$ nucleus. The above parameters are obtained at $Q^{2}=20 \mathrm{GeV}^{2}$. Even though the model can explain the data, it is known that the original fits are no longer valid if we include Fermi-motion effects [33]. Furthermore, the rescaling parameters are calculated in a "semi-classical" way in the sense that overlapping volumes are estimated simply by the geometrical ones. Considering these points, we do not think that the parameters are not well defined in their overall magnitudes. According to the dynamical rescaling model by Close, Roberts, and Ross [2] in 1988, nuclear dependence is the result of a scale change. So, we could in principle set a rescaling parameter by fitting $F_{2}^{A}(x)$ data of a medium-size nucleus, and then use the density dependence of the rescaling model in calculating $\xi_{A}$ for other nuclei. However, we find in our numerical analysis that the above $\xi_{A}$ explain the SLAC data reasonably well, so that we simply keep the original rescaling parameters in Table II of Ref. 2.

We discuss results for the calcium nucleus in Figs. 4a and 4b. In Fig. 4a, theoretical results in Eqs. (5) and (7) are compared with the SLAC data [28] in the linear $x$ scale. For simplicity, the deuteron structure functions are assumed as $F_{2}^{D}(x)=\left[F_{2}^{p}(x)+F_{2}^{n}(x)\right] / 2 \equiv$ $F_{2}^{N}(x)$ in our theoretical analysis. Two sets of results are shown: the solid curves are obtained by using the MRS-1 distributions [31] and the dotted curves are by the KMRSB0 [32]. A, B, C, and $\mathrm{C}^{\prime}$ in the figure show

$$
\begin{array}{ll}
(A) \quad\left[F_{2}^{N}\left(x, Q^{2}\right)+\Delta F_{2}\left(x, Q^{2}\right)\right] / F_{2}^{N}\left(x, Q^{2}\right), \\
(B) \quad\left[F_{2}^{N}\left(x, \xi_{A} Q^{2}\right)+\Delta F_{2}\left(x, Q^{2}\right)\right] / F_{2}^{N}\left(x, Q^{2}\right), \\
(C) \quad\left[F_{2}^{N}\left(x, Q^{2}\right)+\Delta F_{2}\left(x, Q^{2}\right)+\delta F_{2}\left(x, Q^{2}\right)\right] / F_{2}^{N}\left(x, Q^{2}\right), \\
\left(C^{\prime}\right) \quad\left[F_{2}^{N}\left(x, \xi_{A} Q^{2}\right)+\Delta \tilde{F}_{2}\left(x, Q^{2}\right)+\delta \tilde{F}_{2}\left(x, Q^{2}\right)\right] / F_{2}^{N}\left(x, Q^{2}\right),
\end{array}
$$

where $\Delta \tilde{F}_{2}\left(x, Q^{2}\right)$ and $\delta \tilde{F}_{2}\left(x, Q^{2}\right)$ are recombination results with $Q^{2}$-rescaled input distributions. Direct recombination contributions, shown by the curves with A, are positive 
in the medium and large $x$ regions. These come from the quark-gluon fusion processes in Figs. 1a and 1d. Combined results with the $Q^{2}$ rescaling are shown by the curves with B. The $\mathrm{C}$ and $\mathrm{C}^{\prime}$ curves are explained in the following paragraph. Fig. 4a indicates that our model results are in overall agreement with the experimental data. The EMC effect in the medium $x$ region is mainly due to the $Q^{2}$ rescaling in our model, because the recombination contributions are rather small. It is noteworthy in these figures that the recombination can explain nuclear structure functions in the large $x$ region without explicit Fermi-motion effects. Physics in this region is usually attributed to the nucleon Fermi motion in the nucleus. In our model, a quark with $x_{\sim}^{<} 1$ could be pushed by a gluon from a different nucleon and becomes a quark with $x>1$. This is the reason why the ratio goes to infinity at $x \rightarrow 1$ in Fig. 4a. Perhaps, there exists a correlation between these two apparently different descriptions: the parton recombination and the nucleon Fermi motion.

In Fig. 4b, theoretical results are compared with EMC [10] and NMC [8] data in the logarithmic $x$ scale. In the small $x$ region, the processes in Figs. 1b, 1c, 1e, and $1 \mathrm{f}$ significantly contribute and produce negative (shadowing) results in the case of KMRS-B0. However, they are too small to explain the whole shadowing data if the recombinations are calculated at $Q^{2}=5 \mathrm{GeV}^{2}$. The important point in our model is the shadowing which is produced through the gluon distributions. Combined results are shown by the curves with $\mathrm{C}$ or $\mathrm{C}^{\prime}$ in Fig. 4b. The figure indicates that the shadowing produced through modifications in gluon distributions can explain the EMC and NMC shadowing data fairly well, even though the direct recombination effects on $F_{2}(x)$ (shown by $\mathrm{A}$ ) are very small. The curves $\mathrm{C}$ are obtained without rescaling and $\mathrm{C}^{\prime}$ is with the $Q^{2}$-rescaled inputs, where the same $\xi_{A}$ is used in all parton distributions. We find that the differences between the $\mathrm{C}$ and $\mathrm{C}^{\prime}$ curves are rather small considering the fact that the $Q^{2}$ rescaling produces large sea-quark modifications. This is because the $Q^{2}$-rescaled sea-quark distributions and contributions (Eq. (6)) from $Q^{2}$-rescaled gluon distributions almost cancel each other.

We have shown that our model can explain the NMC shadowing in Fig. 4b. However, the results are very sensitive to input gluon distributions in the small $x$ region. Because the E665 collaboration measured $F_{2}^{X e}(x) / F_{2}^{D}(x)$ at very small $x$ recently, we compare our results with the E665 data. In order to find details of input gluon distribution effects, we use parton distributions with "hard", "soft", and " $1 / \sqrt{x}$ " gluon distributions given by the 
MRS [31]. Analytical distributions at $Q^{2}=4 \mathrm{GeV}^{2}$ are used as the input distributions. The MRS soft, hard, and $1 / \sqrt{x}$ gluon distributions are given by $\delta_{G}=0, \eta_{G}=5, \gamma_{G}=0$, $\delta_{G}=0, \eta_{G}=4, \gamma_{G}=9$, and $\delta_{G}=-1 / 2, \eta_{G}=4, \gamma_{G}=9$ respectively for the notation $x G(x)=A_{G} x_{G}^{\delta}(1-x)^{\eta_{G}}\left(1+\gamma_{G} x\right)$. These gluon distributions are shown in Fig. 5a and they are very different especially at $x<0.01$. Our shadowing results are shown in Fig. 5b. Because the experimental $Q^{2}$ of the E665 data becomes very small $\left(Q^{2}<<1 \mathrm{GeV}^{2}\right)$ at $x<0.001$, our perturbative results cannot be compared with the E665 data. This is the reason why curves in the region $x<0.001$ are shown by the dashed ones. As shown in the figure, our calculations produce widely-different shadowing results depending on the input gluon distribution. If it is the $1 / \sqrt{x}$ distribution, shadowings are too large and are contradictory to the experimental data. If it is the hard one, theoretical results underestimate the shadowing. Our theoretical results are nearly consistent with experimental data if the input distributions are the soft MRS distributions at $Q^{2}=4 \mathrm{GeV}^{2}$.

We found that our model with the recombination and the rescaling works in the wide$x$ region, if an appropriate input gluon distribution is taken. Nevertheless, it is not a complete description in the sense that $F_{2}^{A}(x)$ in the $x \sim 0.1$ region cannot be calculated. Furthermore, a curve in the $x>0.2$ region and one in the $x<0.1$ are not smoothly connected in Fig. 4b. It implies that the analytical solution Eq. (6) at $x<0.1$ is used beyond the applicable region. In order to have detailed comparisons with experimental data and to have a dynamically consistent picture, $Q^{2}$ evolution effects should be fully investigated. We address this point in the following section. 


\section{4. $\mathrm{Q}^{2}$ evolution of nuclear structure functions}

\section{1 $\mathrm{Q}^{2}$ evolution}

The approximate description with Eq. (6) is good enough for rough magnitude estimates. For detailed comparisons with the experimental data in the $x \approx 0.1$ region, we should solve $Q^{2}$-evolution equations. Namely, we calculate the recombination and the rescaling at small $Q^{2}\left(\equiv Q_{0}^{2}\right)$, then obtained distributions are evolved to those at larger $Q^{2}$ where experimental data are taken. However, this is not an easy work by the following reasons.

First, the shadowing data are mostly taken in the $Q^{2}$ region of a few $\mathrm{GeV}^{2}$. We need to assign a very small value to $Q_{0}^{2}$, for example $Q_{0}^{2}=1 \mathrm{GeV}^{2}$, in order to study evolution effects by comparing our results with the existing data. The perturbative QCD, especially in the leading order, would become questionable in such small $Q^{2}$ region. Nevertheless, we present the evolution effects in this report because the gluon shadowing connected with the $Q^{2}$ evolution is the essential part of our model in explaining the $F_{2}$ shadowing, as we found in the previous section. Next-to-leading (and higher) order corrections are important in the small $Q^{2}$ and small $x$ region. Hence, our investigation should be considered as a first step for understanding $F_{2}^{A}(x)$ dynamically in the wide $x$ region within the framework of a quark-parton model.

Secondly, the evolution equation for parton distributions in a nucleus is given in Ref. 13. Finding a numerical solution for the equation is by itself a significant research problem. So, instead of solving the nuclear evolution equation, we use the Altarelli-Parisi equation for our $Q^{2}$ evolution. Both evolution results are qualitatively similar; however, the nuclear-evolution shows smaller $Q^{2}$-dependence for large nuclei [13]. Because our results indicate small $Q^{2}$ dependence for large nuclei in section 4.2, this problem is not considered to be very serious.

We calculate $F_{2}^{A}(x)$ in the following way. Valence-quark distributions in the nucleon are modified according to the $Q^{2}$ rescaling mechanism at $Q_{0}^{2}: \tilde{V}\left(x, Q_{0}^{2}\right)=V\left(x, \xi_{A}^{V} Q_{0}^{2}\right)$. In order to satisfy the momentum conservation $\int d x x\left[\tilde{u}_{v}(x)+\tilde{d}_{v}(x)+\tilde{S}(x)+\tilde{G}(x)\right]=1$, sea-quark and gluon distributions are simply modified as $\tilde{S}\left(x, Q_{0}{ }^{2}\right)=C_{s g} S\left(x, Q_{0}{ }^{2}\right)$ and $\tilde{G}\left(x, Q_{0}^{2}\right)=C_{s g} G\left(x, Q_{0}^{2}\right)$. (It is not clear whether the rescaling picture could be used 
also for sea-quarks and gluons. This issue is discussed in section 4.3.) Obtained parton distributions are then used for calculating the recombination effects in Appendix, and we get nuclear parton distributions at $Q_{0}^{2}$. These distributions are evolved to those at larger $Q^{2}$ by using the ordinary Altarelli-Parisi equation [24]. For the flavor-singlet case, it is

$$
\begin{aligned}
\frac{\partial}{\partial t} q_{s}(x, t) & =\int_{x}^{1} \frac{d y}{y}\left[q_{s}(y, t) P_{q q}\left(\frac{x}{y}\right)+G(y, t) P_{q G}\left(\frac{x}{y}\right)\right] \\
\frac{\partial}{\partial t} G(x, t) & =\int_{x}^{1} \frac{d y}{y}\left[q_{s}(y, t) P_{G q}\left(\frac{x}{y}\right)+G(y, t) P_{G G}\left(\frac{x}{y}\right)\right]
\end{aligned}
$$

where $q_{s}$ is the flavor-singlet distribution given by $q_{s}=\sum_{i}\left(q_{i}+\bar{q}_{i}\right), P_{i j}$ is the splitting function, and $t$ is defined by $t=-\left(2 / \beta_{0}\right) \ln \left[\alpha_{s}\left(Q^{2}\right) / \alpha_{s}\left(Q_{0}^{2}\right)\right]$. We used a solution of this integrodifferential equation in the leading order [34]. In the flavor-nonsinglet case, the gluon does not enter into the evolution equation:

$$
\frac{\partial}{\partial t} q_{n s}(x, t)=\int_{x}^{1} \frac{d y}{y} q_{n s}(y, t) P_{q q}\left(\frac{x}{y}\right)
$$

where $q_{n s}=q_{i}-\bar{q}_{i}$. We use the distributions at $Q_{0}^{2}$ calculated by the rescaling and the recombinations in the above equations: $q_{n s}\left(x, Q_{0}^{2}\right)=\tilde{u}_{v}\left(x, Q_{0}^{2}\right)+\tilde{d}_{v}\left(x, Q_{0}^{2}\right)$, $q_{s}\left(x, Q_{0}^{2}\right)=\tilde{u}_{v}\left(x, Q_{0}^{2}\right)+\tilde{d}_{v}\left(x, Q_{0}^{2}\right)+\tilde{S}\left(x, Q_{0}^{2}\right)$, and $G\left(x, Q_{0}^{2}\right)=\tilde{G}\left(x, Q_{0}^{2}\right)$. We then obtain evolved distributions by solving the above equations, and $F_{2}$ at $Q^{2}$ is given by $F_{2}\left(x, Q^{2}\right)=\left[x q_{n s}\left(x, Q^{2}\right)+4 x q_{s}\left(x, Q^{2}\right)\right] / 18$. The use of the above equations instead of the nuclear evolution equations causes a problem in the large $x$ region, because the recombinations produce distributions at $x>1$. However, it is not a serious problem in comparison with the experimental data discussed in the next section.

\section{2 $Q^{2}$ evolution results}

Structure functions $F_{2}^{A}(x)$ are calculated in the following way. Because the parton distributions in the MRS have simpler analytical forms than those in the KMRS, we first employ the MRS-1 (soft gluon) as our parton distributions in the nucleon. Using the 
analytical form of the MRS-1 at $Q^{2}=4 \mathrm{GeV}^{2}$ and the evolution subroutine in Ref. 34, we calculate distributions at $Q_{0}^{2}\left(\approx 1 \mathrm{GeV}^{2}\right)$. Then, the $Q^{2}$ rescaling is used for valence distributions and the constant $C_{s g}$ is determined so that the momentum conservation is satisfied. Input distributions at $Q_{0}^{2}$ are parametrized by a simple analytical form, $x p(x)=A x^{\delta}(1-x)^{\eta}(1+\gamma x)$. Constants $A, \delta, \eta$, and $\gamma$ are obtained by fitting numerical results. The recombinations are calculated by using the modified input distributions at $Q_{0}^{2}$, and obtained results are again fitted by $x p(x)=A x^{\delta}(1-x)^{\eta}(1+\gamma x)$. For the valence quarks, we used $x V(x)=A x^{\delta}(1-\beta x)^{\eta}(1+\gamma x)$, where $\beta<1$, so that we could take into account the fusion effects at large $x(=0.8-0.9)$. The recombinations produce distributions at $x>1$; however, we simply neglected such distributions in our $Q^{2}$ evolution. This causes slight inconsistency, but the neglected effect on the momentum conservation is of the order of $10^{-4} \%$, which is insignificant.

We discuss results for the nuclei $\mathrm{He}, \mathrm{C}, \mathrm{Ca}, \mathrm{Ag}$, $\mathrm{Sn}$, and Xe in Figs. 6-9. In Figs. 6a-9a, our theoretical results are compared with SLAC [28] and EMC [29] (E665 [9]) data in the linear $x$ scale. In order to see the shadowing due to the $Q^{2}$ evolution, we should choose $Q^{2} \sim 1 \mathrm{GeV}^{2}$ because the data were taken in the region of a few $\mathrm{GeV}^{2}$. The value of $Q_{0}^{2}$ is adjusted so that our results agree with the shadowing data for ${ }^{40} \mathrm{Ca}$, and we obtain $Q_{0}^{2}=0.8 \mathrm{GeV}^{2}$. We fixed the cutoff at $z_{0}=2 \mathrm{fm}$ as it was used in section 3 . We discuss dependence of our results on these parameters in the next section. Another problem is how we should choose the rescaling parameters at very small $Q^{2}$. As we did in section 3 , we may simply determine $\xi_{A}$ for a medium-size nucleus and then use the A-dependence for other nuclei. However, it turns out that an original rescaling parameter for a medium-size nucleus is not a bad choice, so we simply use the parameters in Ref. 2. Three curves with $Q^{2}=0.8,5.0,20.0 \mathrm{GeV}^{2}$ are shown in Figs. 6-9. Figures 6a-9a indicate that our model can explain the experimental data well except for the $x \approx 0.7$ region of large nuclei. The results in this region depend much on the input valence distribution and the cutoff (see section 4.3). If we change the input to the KMRS-B0 distributions, our calculations work very well for large nuclei, but the EMC effect for ${ }^{4} \mathrm{He}$ is overestimated.

In Figs. 6b-9b, theoretical results are compared with EMC [10], NMC [8], and E665 [9] data in the logarithmic $x$ scale. Our results are not shown at $x<0.001$ in Fig. 9b because the used evolution subroutine [34] does not have good accuracy in such region and 
our perturbative calculations cannot be compared with the data with very small $Q^{2}$. We find in Figs. 6b-9b that our results agree quite well with the experimental data. In the small nuclei (Figs. 6b and 7b), there are some $Q^{2}$ dependence at $Q^{2}=0.8-2 \mathrm{GeV}^{2}$, but it becomes $Q^{2}$ independent at $Q^{2}>$ a few $\mathrm{GeV}^{2}$. We have very small $Q^{2}$ dependence in the larger nuclei as shown in Figs. $8 \mathrm{~b}$ and $9 \mathrm{~b}$. We note that rather large $Q^{2}$ dependence in Figs. $6 \mathrm{~b}$ and $7 \mathrm{~b}$ should not be taken seriously at this stage, because small variations in input sea-quark and gluon distributions change the dependence drastically as we find in section 4.3 .

\subsection{Dependence on the parameters}

We discuss how our results depend on the parameters, the input distributions, and the rescaling assumption. We first check the choice of $Q_{0}{ }^{2}$. We choose $Q_{0}{ }^{2}=2.0 \mathrm{GeV}^{2}$ instead of $0.8 \mathrm{GeV}^{2}$ in Fig. 10. Distributions at $2 \mathrm{GeV}^{2}$ are calculated and are then evolved to those at 5 and $20 \mathrm{GeV}^{2}$. It is obvious that our calculations underestimate the experimental shadowings and that the $Q^{2}$ dependence is very different from the one in Fig. $8 \mathrm{~b}$. The differences are due to the $Q^{2}$ factor in $K$ (see Eq. (1)) and due to input sea-quark and gluon distributions. In fact, we find in the following that slight modifications in those distributions change our shadowing results at $Q_{0}^{2}$ significantly.

In order to check input-distribution effects, we take the KMRS-B0 distributions which are slightly different from those of the MRS-1. These distributions are shown in Fig. 11. Even though two distributions do not look very different, $Q^{2}$ dependency of the KMRS-B0 results for the carbon nucleus in Fig. 12a is quite different from that in Fig. 7b. The KMRS-B0 results for the calcium in Fig. 12b are rather similar to those of the MRS-1 in Fig. 8b. Because the parton distributions in the small- $x$ region are not well known, we inevitably have such uncertainty in our model.

Effects of a momentum cutoff for leak-out partons are shown in Figs. 13a and 13b. From Fig. 3, we decided to take $z_{0}=2 \mathrm{fm}$ and we have been using this cutoff so far. Results with $z_{0}=3 \mathrm{fm}$ are shown by the dashed curves. Our model with larger $z_{0}$ produces smaller recombination effects at large $x$ and larger effects at small $x$. The agreement with the data becomes better in Fig. 13a in the medium- $x$ region; however, it becomes worse in 
the small- $x$ region in Fig. 13b. Because the cutoff is one of the important factors in our model, efforts should be made to estimate it in an independent way.

The last issue is the rescaling. The original rescaling model is intended for valence quarks. It is not clear whether sea-quarks and gluon follow the same rule of the scale change in nuclei. In our analysis of this section, we used the rescaling only for valence quarks. Sea-quark and gluon distributions are simply increased by a constant amount $\left(C_{s g}\right)$ so that they carry a momentum deficit produced by the valence rescaling. This is an arbitrary assumption. We may choose other scenario, for example the $Q^{2}$ rescaling for all partons. Using this rescaling picture, we obtain the results in Fig. 14. The shadowings are much underestimated in this case. Although numerical values do not agree completely with those in Fig. 4b, we find a similar tendency in the sense that calculated shadowings become smaller in the $x$ region $(0.01<x<0.1)$ if the rescaling is used for all partons.

We learned the following from the above analyses. There are a few parameters and assumptions in our model and obtained results depend inevitably on their choice. The major factors are $(1) Q_{0}^{2},(2)$ input $\mathrm{S}(\mathrm{x})$ and $\mathrm{G}(\mathrm{x})$ in the small $x,(3) z_{0}$, and (4) $Q^{2}$ rescaling for (valence) partons. We find that magnitude of our shadowing and the $Q^{2}$ dependence are very sensitive to the above factors. Nevertheless, we find that it is possible to choose a set of the factors within our initial-expectation ranges (e.g. $Q^{2} \sim 1 \mathrm{GeV}^{2}$, $z_{0} \sim 2 \mathrm{fm}$ ) and we explain many existing data. Obviously, much efforts should be done for investigating these factors and also next-to-leading order effects.

\section{Conclusions}

We investigated nuclear structure functions $F_{2}\left(x, Q^{2}\right)$ from small $x\left(\approx 10^{-3}\right)$ to large $x(\approx 0.9)$ in a parton model based on a $Q^{2}$ rescaling model with parton recombination effects in order to compare them with the recent experimental data. As a result, we obtained reasonably good agreement with the experimental data in the region $(0.005<$ $x<0.8)$. In the large $x$ region, the ratio $\left(F_{2}^{A}(x) / F_{2}^{D}(x)>1\right)$ is explained by quark-gluon recombinations, which produce results similar to those by the nucleon Fermi motion. In the medium $x$ region, the EMC effect is mainly due to the $Q^{2}$ rescaling mechanism in our model. In the small $x$ region, shadowing effects are obtained through modifications in 
gluon distributions. However, our shadowings at very small $x(<0.02)$ are very sensitive to the input gluon distribution. We have a few parameters in our model; however, we could choose a set of the parameters and explain many existing experimental data. $Q^{2}$ variations of our shadowing results depend much on the input sea-quark and gluon distributions, and also on the parameters.

\section{Acknowledgments}

This research was supported by the Deutsche Forschungsgemeinschaft (SFB 201) and by the US-NSF under Contract No. NSF-PHY91-08036. S.K. thanks Drs. F. E. Close, J. Qiu, and R. G. Roberts for helpful suggestions and for patiently answering questions about parton recombinations; Drs. M. van der Heijden, Q. Ingram, and G. van Middelkoop for discussion about NMC experiments; Drs. C. W. Salgado and H. M. Schellman for information about the E665 experimental results; Dr. W. J. Stirling for sending their computer programs for calculating the parton distributions in Ref. 32 . 


\section{Appendix}

Although detailed formalisms are given in Ref. 17, explicit equations used in this investigation are shown in the following. Using Eqs. (1)-(4) and changing the integration variable $x_{1} \leftrightarrow x_{2}$ for the process in Fig. 1e, we obtain modifications of quark distributions in nuclei due to the parton recombination mechanism:

$$
\begin{aligned}
x \cdot \Delta q_{i}(x)= & +\frac{K}{6} \int_{0}^{x} \frac{d x_{2}}{x_{2}} x_{2} G^{*}\left(x_{2}\right)\left[\left(x-x_{2}\right) q_{i}\left(x-x_{2}\right)\left\{1+\left(\frac{x-x_{2}}{x}\right)^{2}\right\}\right. \\
& \left.-x q_{i}(x) \frac{x}{x+x_{2}}\left\{1+\left(\frac{x}{x+x_{2}}\right)^{2}\right\}\right] \\
& -\frac{K}{6} \int_{x}^{1} \frac{d x_{2}}{x_{2}} x q_{i}(x) x_{2} G^{*}\left(x_{2}\right) \frac{x}{x+x_{2}}\left\{1+\left(\frac{x}{x+x_{2}}\right)^{2}\right\} \\
& -\frac{4 K}{9} x \int_{0}^{1} d x_{2} x q_{i}(x) x_{2} \bar{q}_{i}^{*}\left(x_{2}\right) \frac{x^{2}+x_{2}^{2}}{\left(x+x_{2}\right)^{4}} \\
& +\frac{K}{6} \int_{0}^{x} \frac{d x_{1}}{x_{1}} x_{1} G\left(x_{1}\right)\left[\left(x-x_{1}\right) q_{i}^{*}\left(x-x_{1}\right)\left\{1+\left(\frac{x-x_{1}}{x}\right)^{2}\right\}\right. \\
& -\frac{K}{6} \int_{x}^{1} \frac{d x_{1}}{x_{1}} x_{1} G\left(x_{1}\right) x q_{i}^{*}(x) \frac{x}{x+x_{1}}\left\{1+\left(\frac{x}{x+x_{1}}\right)^{2}\right\} \\
& -\frac{4 K}{9} x \int_{0}^{1} d x_{2} x q_{i}^{*}(x) x_{2} \bar{q}_{i}\left(x_{2}\right) \frac{x^{2}+x_{2}^{2}}{\left(x+x_{2}\right)^{4}},
\end{aligned}
$$

where $*$ indicates a leak-out parton. For example a gluon, which leaks out from the nucleon 2 , interacts with a quark in the nucleon 1 and produces a quark with momentum $x$ in the first term of Eq. (A1). Explicit $Q^{2}$ dependence in parton distributions is not shown in this appendix in order to simplify the notations. Modifications in antiquark distributions are obtained in the similar way by changing $q_{i} \leftrightarrow \bar{q}_{i}$ in Eq. (A1). Using the above 
expression, we obtain parton recombination effects on the structure functions $F_{2}(x)$ by $\Delta F_{2}(x)=\sum_{i} e_{i}^{2} x\left[\Delta q_{i}(x)+\Delta \bar{q}_{i}(x)\right]$

$\Delta F_{2}(x)=\Delta F_{2}^{(1)}(x)+\Delta F_{2}^{(2)}(x)+\Delta F_{2}^{(3)}(x)$

$\Delta F_{2}^{(1)}(x)=+\frac{K}{6} \int_{0}^{x} \frac{d x_{2}}{x_{2}} x_{2} G^{*}\left(x_{2}\right)\left[F_{2}\left(x-x_{2}\right)\left\{1+\left(\frac{x-x_{2}}{x}\right)^{2}\right\}\right.$

$\left.-F_{2}(x) \frac{x}{x+x_{2}}\left\{1+\left(\frac{x}{x+x_{2}}\right)^{2}\right\}\right]$

$-\frac{K}{6} \int_{x}^{1} \frac{d x_{2}}{x_{2}} F_{2}(x) x_{2} G^{*}\left(x_{2}\right) \frac{x}{x+x_{2}}\left\{1+\left(\frac{x}{x+x_{2}}\right)^{2}\right\}$

$\Delta F_{2}^{(2)}(x)=+\frac{K}{6} \int_{0}^{x} \frac{d x_{1}}{x_{1}} x_{1} G\left(x_{1}\right)\left[\sum_{i} e_{i}^{2}\left\{\left(x-x_{1}\right) q_{i}^{*}\left(x-x_{1}\right)+\left(x-x_{1}\right) \bar{q}_{i}^{*}\left(x-x_{1}\right)\right\}\right.$

$$
\times\left\{1+\left(\frac{x-x_{1}}{x}\right)^{2}\right\}
$$

$\left.-\sum_{i} e_{i}^{2}\left\{x q_{i}^{*}(x)+x \bar{q}_{i}^{*}(x)\right\} \frac{x}{x+x_{1}}\left\{1+\left(\frac{x}{x+x_{1}}\right)^{2}\right\}\right]$

$-\frac{K}{6} \int_{x}^{1} \frac{d x_{1}}{x_{1}} x_{1} G\left(x_{1}\right) \sum_{i} e_{i}^{2}\left\{x q_{i}^{*}(x)+x \bar{q}_{i}^{*}(x)\right\} \frac{x}{x+x_{1}}\left\{1+\left(\frac{x}{x+x_{1}}\right)^{2}\right\}$

$\Delta F_{2}^{(3)}(x)=-\frac{4 K}{9} x \int_{0}^{1} d x_{2} \sum_{i} e_{i}^{2}\left\{x q_{i}(x) x_{2} \bar{q}_{i}^{*}\left(x_{2}\right)+x \bar{q}_{i}(x) x_{2} q_{i}^{*}\left(x_{2}\right)\right\} \frac{x^{2}+x_{2}^{2}}{\left(x+x_{2}\right)^{4}}$

$-\frac{4 K}{9} x \int_{0}^{1} d x_{2} \sum_{i}^{-} e_{i}^{2}\left\{x q_{i}^{*}(x) x_{2} \bar{q}_{i}\left(x_{2}\right)+x \bar{q}_{i}^{*}(x) x_{2} q_{i}\left(x_{2}\right)\right\} \frac{x^{2}+x_{2}^{2}}{\left(x+x_{2}\right)^{4}}$

where $\bar{\sum}$ indicates that the summation is averaged over partons in the proton and the neutron. For example, they are $\sum_{i} e_{i}^{2} q_{i}=\left[\frac{5}{18}(u+d)+\frac{2}{18} s\right]_{\text {proton }}$ and $\sum_{i} e_{i}^{2} q_{i} \bar{q}_{i}=$ 
$\left[\frac{5}{18}(u \bar{u}+d \bar{d})+\frac{2}{18} s \bar{s}\right]_{\text {proton }}$.

In the similar way, modifications in a gluon distribution in a nucleus are obtained as $[35]$

$x \Delta G(x)=x \Delta G^{(1)}(x)+x \Delta G^{(2)}(x)+x \Delta G^{(3)}(x)$

$$
\begin{aligned}
x \Delta G^{(1)}(x)= & +\frac{3 K}{4} x \int_{0}^{x} d x_{1} x_{1} G\left(x_{1}\right)\left(x-x_{1}\right) G^{*}\left(x-x_{1}\right) \\
& \times \frac{1}{x^{2}}\left\{\frac{x_{1}}{x-x_{1}}+\frac{x-x_{1}}{x_{1}}+\frac{x_{1}\left(x-x_{1}\right)}{x^{2}}\right\} \\
& -\frac{3 K}{4} x \int_{0}^{1} d x_{2} x G(x) x_{2} G^{*}\left(x_{2}\right) \frac{1}{\left(x+x_{2}\right)^{2}}\left\{\frac{x}{x_{2}}+\frac{x_{2}}{x}+\frac{x x_{2}}{\left(x+x_{2}\right)^{2}}\right\} \\
& -\frac{3 K}{4} x \int_{0}^{1} d x_{2} x G^{*}(x) x_{2} G\left(x_{2}\right) \frac{1}{\left(x+x_{2}\right)^{2}}\left\{\frac{x}{x_{2}}+\frac{x_{2}}{x}+\frac{x_{2}}{\left(x+x_{2}\right)^{2}}\right\} \\
x \Delta G^{(2)}(x)= & +\frac{4 K}{9} x \int_{0}^{x} d x_{1} \sum_{i}\left\{x_{1} q_{i}\left(x_{1}\right)\left(x-x_{1}\right) \bar{q}_{i}^{*}\left(x-x_{1}\right)\right. \\
& \left.+x_{1} \bar{q}_{i}\left(x_{1}\right)\left(x-x_{1}\right) q_{i}^{*}\left(x-x_{1}\right)\right\} \frac{1}{x^{4}}\left\{x_{1}^{2}+\left(x-x_{1}\right)^{2}\right\}
\end{aligned}
$$

$$
\begin{aligned}
x \Delta G^{(3)}(x)= & -\frac{K}{6} \int_{0}^{1} d x_{2} x G(x) \sum_{i}\left\{x_{2} q_{i}^{*}\left(x_{2}\right)+x_{2} \bar{q}_{i}^{*}\left(x_{2}\right)\right\} \frac{1}{x+x_{2}}\left\{1+\left(\frac{x_{2}}{x+x_{2}}\right)^{2}\right\} \\
& -\frac{K}{6} \int_{0}^{1} d x_{2} x G^{*}(x) \sum_{i}\left\{x_{2} q_{i}\left(x_{2}\right)+x_{2} \bar{q}_{i}\left(x_{2}\right)\right\} \frac{1}{x+x_{2}}\left\{1+\left(\frac{x_{2}}{x+x_{2}}\right)^{2}\right\}
\end{aligned}
$$

where $\sum_{i}^{-} q_{i}=\sum_{i}\left(q_{i}\right)_{\text {proton }}$ and $\sum_{i}^{-} q_{i} \bar{q}_{i}=\sum_{i}\left(q_{i} \bar{q}_{i}\right)_{\text {proton. }}$. Using these expressions, we find that the momentum conservation is explicitly satisfied, $\int d x\left[\sum_{i} x\left\{\Delta q_{i}(x)+\Delta \bar{q}_{i}(x)\right\}\right.$ 
$+x \Delta G(x)]=0$. For numerical analysis, the last integral in $\Delta G^{(1)}(x)$ should be evaluated by separating the integral region $\int_{0}^{1} d x_{2}=\int_{0}^{x} d x_{2}+\int_{x}^{1} d x_{2}$ and by changing variables $x^{\prime}=x-x_{2}$ in the integral $\int_{0}^{x} d x_{2}$. We use the following equations for evaluating $\Delta G^{(1)}(x)$ in order to cancel out infinities:

$$
\begin{aligned}
& x \Delta G^{(1)}(x)= \int_{0}^{x / 2} d x^{\prime}\left[f\left(x, x^{\prime}\right)+f\left(x, x-x^{\prime}\right)\right]-\int_{x}^{1} d x^{\prime} g\left(x, x^{\prime}\right) \\
& f\left(x, x^{\prime}\right)=+\frac{3 K}{4} x x^{\prime} G\left(x^{\prime}\right)\left(x-x^{\prime}\right) G^{*}\left(x-x^{\prime}\right) \frac{1}{x^{2}}\left\{\frac{x^{\prime}}{x-x^{\prime}}+\frac{x-x^{\prime}}{x^{\prime}}+\frac{x^{\prime}\left(x-x^{\prime}\right)}{x^{2}}\right\} \\
&-\frac{3 K}{4} x \quad x G(x) x^{\prime} G^{*}\left(x^{\prime}\right) \frac{1}{\left(x+x^{\prime}\right)^{2}}\left\{\frac{x}{x^{\prime}}+\frac{x^{\prime}}{x}+\frac{x x^{\prime}}{\left(x+x^{\prime}\right)^{2}}\right\} \\
&- \frac{3 K}{4} x \quad x G^{*}(x)\left(x-x^{\prime}\right) G\left(x-x^{\prime}\right) \frac{1}{\left(2 x-x^{\prime}\right)^{2}}\left\{\frac{x}{x-x^{\prime}}+\frac{x-x^{\prime}}{x}+\frac{x\left(x-x^{\prime}\right)}{\left(2 x-x^{\prime}\right)^{2}}\right\} \\
& g\left(x, x^{\prime}\right)=+\frac{3 K}{4} x\left[x G(x) x^{\prime} G^{*}\left(x^{\prime}\right)+x G^{*}(x) x^{\prime} G\left(x^{\prime}\right)\right] \\
& \times \frac{1}{\left(x+x^{\prime}\right)^{2}}\left\{\frac{x}{x^{\prime}}+\frac{x^{\prime}}{x}+\frac{x x^{\prime}}{\left(x+x^{\prime}\right)^{2}}\right\}
\end{aligned}
$$

Nuclear gluon distributions in our model are discussed in Ref. 36 and they are compared with recent NMC measurements of $G_{S n}(x) / G_{C}(x)$. 


\section{References}

1. J. J. Aubert et al. (EMC collaboration), Phys. Lett. 123B, 275 (1983).

2. F. E. Close, R. G. Roberts, and G. G. Ross, Phys. Lett. 129B, 346 (1983); Nucl. Phys. B296, 582 (1988); F. E. Close, R. L. Jaffe, R. G. Roberts, and G. G. Ross, Phys. Rev. D31, 1004 (1985).

3. E. L. Berger and F. Coester, Annu. Rev. Nucl. Part. Sci. 37, 463 (1987); R. P. Bickerstaff and A. W. Thomas, J. Phys. G 15, 1523 (1989); R. P. Bickerstaff, M. C. Birse, and G. A. Miller, Phys. Rev. D33, 3228 (1986).

4. D. M. Alde et al. (E772 collaboration), Phys. Rev. Lett. 64, 2479 (1990); A. Magnon, talk given at the workshop on Baryon Spectroscopy and the Structure of the Nucleon, Saclay, France, Sept. 23-25, 1991.

5. S. Kumano and F. E. Close, Phys. Rev. C41, 1855 (1990).

6. S. Kumano, Indiana University preprint IU/NTC-92-02; talk given at the 13th International Conference on Few Body Problems in Physics, Adelaide, Australia, Jan. 5-11, 1992; in Proceedings of the International Workshop on Gross Properties of Nuclei and Nuclear Excitations, Hirschegg, Austria, Jan. 20-25, 1992, edited by H. Feldmeier; to be submitted for publication.

7. T. de Forest and P. J. Mulders, Phys. Rev. D35, 2849 (1987); S. Kumano and E. J. Moniz, Phys. Rev. C37, 2088 (1988).

8. P. Amaudruz et al. (NMC collaboration), Z. Phys. C51, 387 (1991); M. van der Heijden, Ph.D thesis, University of Amsterdam (1991); M. A. J. Botje and C. Scholz in Intersections between Particle and Nuclear Physics, edited by W. T. H. van Oers, American Institute of Physics (1991).

9. M. R. Adams et al. (E665 collaboration), Phys. Rev. Lett. 68, 3266 (1992); D. E. Jaffe, in Intersections between Particle and Nuclear Physics, edited by W. T. H. van Oers, American Institute of Physics (1991); C. W. Salgado, talk given at the workshop on High Energy Probes of QCD and Nuclei, University Park, Pennsylvania, March 25-28, 1992. 
10. M. Arneodo et al. (EMC collaboration), Nucl. Phys. B333, 1 (1990); Phys. Lett. 211B, 493 (1988).

11. For recent investigations on the vector meson dominance model, see C. L. Bilchak, D. Schildknecht, and J. D. Stroughair, Phys. Lett. 214B, 441 (1988); 233B, 461 (1989); G. Shaw, Phys. Lett. 228B, 125 (1989); G. Piller and W. Weise, Phys. Rev. C42, R1834 (1990).

12. N. N. Nicolaev and V. I. Zakharov, Phys. Lett. 55B, 397 (1975).

13. A. H. Mueller and J. Qiu, Nucl. Phys. B268, 427 (1986); J. Qiu, Nucl. Phys. B291, 746 (1987); E. L. Berger and J. Qiu, Phys. Lett. 206B, 141 (1988).

14. F. E. Close and R. G. Roberts, Phys. Lett. 213B, 91 (1988).

15. R. J. M. Covolan and E. Predazzi, Nuovo Cimento, 103A, 773 (1990); W. Zhu and J. G. Shen, Phys. Lett. 235B, 170 (1990); J. Phys. G16, 925 (1990); G. Li, Z. Cao, and C. Zhong, Nucl. Phys. A509, 757 (1990); M. Altmann, M. Glück, and E. Reya, Phys. Lett. 285B, 359 (1992).

16. L. L. Frankfurt, S. Liuti, and M. I. Strikman, research in progress; S. Liuti, personal communication; V. Barone et al., preprint KFA-IKP(TH)-1992-13.

17. F. E. Close, J. Qiu, and R. G. Roberts, Phys. Rev. D40, 2820 (1989).

18. L. L. Frankfurt and M. I. Strikman, Phys. Rep. 160, 235 (1988); Nucl. Phys. B316, 340 (1989); Phys. Rev. Lett. 65, 1725 (1990); S. J. Brodsky and H. J. Lu, Phys. Rev. Lett. 64, 1342 (1990); N. N. Nikolaev and B. G. Zakharov, Phys. Lett. 260B, 414 (1991); Z. Phys. C49, 607 (1991); V. R. Zoller, Z. Phys. C53, 443 (1992); Phys. Lett. 279B, 145 (1992).

19. P. Castorina and A. Donnachie, Phys. Lett. 215B, 589 (1988); Z. Phys. C45, 141 (1989); J. Kwiecinski, Z. Phys. C45, 461 (1990).

20. G. Preparata and P. G. Ratcliffe, preprint MITH91-13.

21. J. Kwiecinski and B. Badelek, Phys. Lett. 208B, 508 (1988); B. Badelek and J. Kwiecinski, Nucl. Phys. B370, 278 (1992); W. Melnitchouk and A. W. Thomas, preprint ADP-92-192-T120. 
22. See Refs. 13 and 17 for obtaining the expression of $K$.

23. R. C. Barrett and D. F. Jackson, Nuclear Sizes and Structure (Clarendon, Oxford, 1977).

24. G. Altarelli and G. Parisi, Nucl. Phys. B126, 298 (1977); In deriving Eq. (2), we should note a factor of 2 coming from averages over initial spin states.

25. F. J. Ynduràin, Quantum Chromodynamics, (Springer-Verlag, New York, 1983).

26. J. P. Ralston, Phys. Lett. 172B, 430 (1986).

27. For a recent summary of experimental data for deep inelastic scatterings, see R. G. Roberts and M. R. Whally, J. Phys. G 17, D1 (1991).

28. R. G. Arnold et al., Phys. Rev. Lett. 52, 727 (1984).

29. J. Ashman et al. (EMC collaboration), Phys. Lett. 202B, 603 (1988).

30. C. H. Llewellyn Smith, Nucl. Phys. A434, 35c (1985).

31. A. D. Martin, R. G. Roberts, and W. J. Stirling, Phys. Rev. D37, 1161 (1988).

32. J. Kwiecinski, A. D. Martin, W. J. Stirling, and R. G. Roberts, Phys. Rev. D42, 3645 (1990).

33. R. P. Bickerstaff and G. A. Miller, Phys. Lett. 168B, 409 (1986).

34. S. Kumano and J. T. Londergan, Comp. Phys. Commu. 69, 373 (1992).

35. J. Qiu, personal communications (1992); Using $K\left[p_{1}\left(x_{1}\right) p_{2}\left(x_{2}\right)+p_{1}\left(x_{2}\right) p_{2}\left(x_{1}\right)\right] \Rightarrow$ $2 K p_{1}\left(x_{1}\right) p_{2}\left(x_{2}\right)$ in Eqs. (A3.3) and (A3.4), we obtain Eq. (24) of Ref. 17 from Eqs. (A3.1)-(A3.4) with $x_{1} \leftrightarrow x_{2}$ in the last integral of Eq. (A3.2) .

36. S. Kumano, Phys. Lett. 298B, 171 (1993); This paper is based on the model in section 3 . 


\section{Figure Captions}

1. Schematic pictures of parton recombination processes.

2. Parton fusions for (a) $q G \rightarrow q$, (b) $q \bar{q} \rightarrow G$, (c) $G q \rightarrow q$, and (d) $G G \rightarrow G$.

3. Momentum cutoff for leak-out partons, $w(x)=\exp \left(-m_{N}^{2} z_{0}^{2} x^{2} / 2\right)$.

4. Comparisons with (a) SLAC data [28], (b) EMC-90 [10] and NMC [8] data for Ca. Solid (dotted) curves are obtained by using the MRS-1 (KMRS-B0) input distributions. $Q^{2}=5 \mathrm{GeV}^{2}$ and $z_{0}=2 \mathrm{fm}$. (A) recombinations, (B) recombinations $+\operatorname{rescaling}\left(\xi_{A}=1.86\right)$. $(\mathrm{C})$ recombinations with gluon-shadowing effects, $\left(\mathrm{C}^{\prime}\right)$ the same as $\mathrm{C}$ except for the rescaling. The only KMRS-B0 curve is shown in $\mathrm{C}^{\prime}$. See text for detailed explanations of $\mathrm{A}, \mathrm{B}, \mathrm{C}$, and $\mathrm{C}^{\prime}$.

5. (a) MRS gluon distributions. See Ref. 31 and text for details of hard, soft, and $1 / \sqrt{x}$ gluon distributions. (b) Comparisons with E665 data for Xe [9] and EMC-88 data for Sn [29]. Theoretical results are obtained (b) for the Xe nucleus $\left(\xi_{A}=2.24\right)$. $Q^{2}=4 \mathrm{GeV}^{2}$ and $z_{0}=2 \mathrm{fm}$.

6. Comparisons with (a) SLAC data [28], (b) NMC data [8] for He. $Q^{2}=0.8,5$, and 20

$\mathrm{GeV}^{2}$ for the dotted, solid, and dashed curves respectively. $z_{0}=2 \mathrm{fm}, \xi_{A}^{V}=1.43$ and $Q_{0}^{2}=0.8 \mathrm{GeV}^{2}$.

7. Comparisons with (a) SLAC [28] and EMC-88 [29] data, (b) EMC-90 [10] and NMC [8] data for C. Notations for the dotted, dashed, and solid curves are the same in Fig. $6 . \xi_{A}^{V}=1.60$.

8. Comparisons with (a) SLAC data [28], (b) EMC-90 [10] and NMC [8] data for Ca. $\xi_{A}^{V}=1.86$.

9. Comparisons with (a) SLAC data for Ag [28], EMC-88 data for Sn [29], and some of E665 data for Xe [9], (b) E665 data for Xe [9] and EMC-88 data for Sn [29]. Calculated results are for $\operatorname{Ag}\left(\xi_{A}^{V}=2.17\right)$ in (a) and for $\mathrm{Xe}\left(\xi_{A}^{V}=2.24\right)$ in (b).

10. Dependence on $Q_{0}{ }^{2} \cdot Q_{0}{ }^{2}=2.0 \mathrm{GeV}^{2}$ is taken. $Q^{2}=2,5$, and $20 \mathrm{GeV}^{2}$ for the dotted, solid, and dashed curves respectively. $z_{0}=2 \mathrm{fm}$ and $\xi_{A}^{V}=1.86$. 
11. Sea-quark and gluon distributions of MRS-1 (solid curves) and KMRS-B0 (dotted curves).

12. Dependence on input distributions (see Fig. 11). Results by using the KMRS-B0 inputs for (a) ${ }^{12} \mathrm{C}$ and (b) ${ }^{40} \mathrm{Ca} . Q^{2}=0.8,5$, and $20 \mathrm{GeV}^{2}$ for the dotted, solid, and dashed curves respectively. $z_{0}=2 \mathrm{fm} . \xi_{A}^{V}=1.60$ for $\mathrm{C}$ and 1.86 for Ca. $Q_{0}^{2}=0.8 \mathrm{GeV}^{2}$. Compare them with the results in Figs. $7 \mathrm{~b}$ and $8 \mathrm{~b}$.

13. Dependence on the cutoff $z_{0}$. Only $Q^{2}=5 \mathrm{GeV}^{2}$ curves are shown. $z_{0}=2.0(3.0) \mathrm{fm}$ for the solid (dashed) curves. $\xi_{A}^{V}=1.86$ and $Q_{0}^{2}=0.8 \mathrm{GeV}^{2}$.

14. Rescaling are used for all partons. Notations and parameters are same in Fig. 7b. 